EU-Aktionsplan: Finanzierung nachhaltigen Wachstums

\section{Das Finanzsystem nachhaltiger gestalten}

\author{
Die EU-Kommission möchte mit einem umfassenden und \\ grundlegenden Maßnahmenpaket die Finanzwirtschaft \\ umgestalten. Wie kann der europäische Finanzsektor öko- \\ logischer und sozialer gestaltet werden? \\ Von Markus Duscha
}

D ie EU-Kommission veröffentlichte am 8. März 2018 - aufbauend auf einem Bericht einer Expertenkommission (HLEG 2018) - den „Aktionsplan: Finanzierung nachhaltigen Wachstums“ (EU-Kommission 2018). Dort wird ein umfangreiches und ambitioniertes Maßnahmenpaket vorgestellt, das darauf abzielt, das Finanzwesen kompatibel zu den Nachhaltigkeitszielen der Europäischen Union (EU) auszugestalten. Der Aktionsplan verfolgt drei zentrale Ziele:

I die Kapitalflüsse auf nachhaltige Investitionen umzulenken,

I finanzielle Risiken, die sich aus der Umweltzerstörung, der Ressourcenknappheit und sozialen Problemen ergeben, zu bewältigen,

I Transparenz und Langfristigkeit in der Finanz- und Wirtschaftstätigkeit $\mathrm{zu}$ fördern.

Bisher gibt es keine einheitliche Regelung, was eine nachhaltige Anlage sein soll. Deshalb betrachtet es die Kommission als vordringliche Maßnahme, ein einheitliches Nachhaltigkeits-Klassifizierungssystem für Finanzprodukte zu entwickeln: Wie lassen sich Umwelt- und soziale Aspekte standardisiert bewerten?

Ein solches Klassifikationssystem soll schließlich Grundlage für verschiedene finanzmarktorientierte politische Instrumente sein, zum Beispiel für Normen, (freiwillige) Kennzeichen, Nachhaltigkeitsbenchmarks und Regulierungsvorschriften. Auch soll untersucht werden, inwiefern die „klassische“ Finanzmarktregulierung mit den Nachhaltigkeitskriterien verbunden werden kann.
In dem Aktionsplan sind auch Maßnahmen zur Förderung von Investitionen in nachhaltige Projekte und zur systematischen Berücksichtigung der Nachhaltigkeitspräferenzen in der Finanzberatung privater Anleger/innen enthalten. Insgesamt wird mit dem Aktionsplan ein grundlegendes und umfassendes Paket skizziert, was noch bis Ende 2019 realisiert werden soll.

\section{Bisherige Reaktionen}

Generell wird die Richtung des Aktionsplans in den bisherigen Reaktionen aus Wirtschaft und Zivilgesellschaft begrüßt. Insbesondere die Notwendigkeit einer einheitlichen Klassifizierung wird durchgängig betont. Bei den darauf aufbauenden Instrumenten gehen die Einschätzungen jedoch naturgemäß auseinander: Während Bankenverbände tendenziell eher eine zu starke Belastung durch weitere Regulierungen wie Berichtspflichten befürchten (z. B. Bundesverband dt. Banken 2018), wünschen sich Umwelt- und Sozialpioniere zum Beispiel verpflichtende statt freiwillige Kennzeichnungen und von vornherein eine stärkere Einbeziehung von weiteren Umwelt- und Sozialkriterien über den Klimaschutz hinaus (CRIC e. V. et al. 2018).

Die von der EU-Kommission geplanten Schritte werden wichtige Grundlagen liefern, um die Finanzbranche verstärkt und endlich in Richtung Nachhaltigkeit zu bewegen. Die hohe beabsichtigte Geschwindigkeit führt jedoch hoffentlich nicht dazu, dass Gründlichkeit und die nötige Tiefe verpasst werden. Zudem erschwert die beabsichtigte Geschwindigkeit insbesondere den lobbymäßig nicht so gut ausgestatteten zivilgesellschaftlichen Gruppierungen, ihre Positionen fundiert und als Gegengewicht der klassischen Finanzbranche einzubringen. Denn es wäre letztlich nicht hilfreich, wenn die Ergebnisse am Ende nicht anspruchsvoll genug sind und dann für lange Zeit niedrige Standards gelten würden.

Deutschland ist im europäischen Vergleich auf diesem Themenfeld bisher kein Vorreiter. Frankreich hat zum Beispiel mit einer Reihe von Maßnahmen hier deutlich innovativere Schritte früher geleistet. Damit sind ausländische Finanzdienstleister zum Teil besser vorbereitet. Hoffentlich wird Deutschland deshalb nicht zum Bremser bei der ambitionierten Umsetzung des Aktionsplans. Es lohnt sich also aus verschiedenen Gründen aktuell sehr, sich für anspruchsvolle Ergebnisse dieses Aktionsplans der EU-Kommission einzusetzen.

\section{Literatur}

Bundesverband deutscher Banken (2018): Deutsche Kreditwirtschaft zum EU-Aktionsplan „Finanzierung nachhaltigen Wachstums“

EU-Kommission (2018): Aktionsplan: Finanzierung nachhaltigen Wachstums.

HLEG (2018): EU High-Level Expert Group on Sustainable Finance: Financing a Sustainable European Economy. Brüssel.

CRIC e. V. et al. (2018): Offener Brief zum Thema Sustainable Finance.

AUTOR + KONTAKT

Markus Duscha arbeitete 25 Jahre für das ifeu - Institut für Energie- und Umweltforschung Heidelberg und gründete 2016 das Fair Finance Institute.

Fair Finance Institute, Montpellierstr. 10, 69115 Heidelberg. Tel.: +496221 4263793, E-Mail: markus.duscha@fair-finance-institute.de 